\title{
OSCILLATIONS OF ROTATING NEUTRON STARS
}

\author{
T. E. STrohmayer \\ Department of Physics and Astronomy, University of Rochester
}

\begin{abstract}
We use a perturbation technique to compute the rotational corrections to the non-radial oscillation spectrum of a realistic neutron-star model. We compute, to first order in the rotation rate, the corrections to the normal mode eigenfrequencies and eigenfunctions. We find that $l=l_{0}$ oscillations are coupled to $l=l_{0} \pm 1$ oscillations by the Coriolis force. For the toroidal modes, this coupling introduces a non-zero radial component to the velocity field. We have used this result to compute the neutrino damping rates for several corrected toroidal modes. This damping mechanism is inoperative for toroidal modes in a non-rotating star because these modes produce no density nor temperature perturbations. The neutrino damping time can approach the gravitational radiation damping time in rotating neutron stars if the central temperature is high enough, $\left(T_{\mathrm{c}} \geq 10^{8} \mathrm{~K}\right)$. The rotationally induced coupling of spheroidal oscillations to toroidal modes can also produce significant displacements at the stellar surface. This may have interesting implications for channeling energy, e.g., that associated with a glitch, to the surface of the star. Perhaps this might produce observable effects in the pulsar emission process or a $\gamma$-ray burst event.
\end{abstract}

\section{Motivation}

The detection of millisecond variability in PSR 2016+28 prompted Boriakoff (1976) and Van Horn (1980) to specuiate that quasi-periodic variability in pulsars is a manifestation of oscillations of the underlying neutron star. Boriakoff suggested that pulsation driven shaking of magnetic field lines "frozen" into the surface layers of the neutron star might modulate the pulsar emission process.

To investigate the neutron star oscillation hypothesis requires coupling a realistic pulsar magnetosphere to an oscillating, magnetic neutron star. Since many magnetosphere models are constructed in the rotating reference frame of the neutron star (Fawley, Arons, and Scharlemann 1977), it is essential that we consider this effect in treating the oscillations. As Carroll et al. (1986) have already conducted a preliminary investigation of the effects of a magnetic field on the oscillations, we devote this paper to a study of the effects of rotation on the oscillation modes of a neutron star.

\section{Oscillation Equations}

The Newtonian hydrodynamic equations valid in a reference frame rotating with uniform angular velocity $\Omega=\Omega \hat{\boldsymbol{z}}$ have the form

$$
\begin{gathered}
\nabla^{2} \Phi=4 \pi G \rho, \\
\frac{\partial \rho}{\partial t}+\nabla \cdot \rho v \cdot=0, \\
\rho\left[\frac{\partial \mathbf{v}}{\partial t}+v \cdot \nabla v+2 \Omega \times v-\Omega^{2} r\right]=\nabla \cdot \vec{\sigma}-\rho \nabla \Phi .
\end{gathered}
$$

Here $\rho$ is the density, $\Phi$ is the gravitational potential, $v=i \sigma \xi$ is the velocity field and $\vec{\sigma}$ is the stress tensor (including non-zero shear terms in the crust).

To investigate small oscillations of the neutron star, we linearize these equations by introducing a set of Eulerian perturbation variables $f^{\prime}(r) e^{i \sigma t}$. Since most pulsars are "slowly rotating," in the sense that the centripetal force due to the stellar rotation is small compared to the gravitational force, we expand the perturbation variables in powers of the rotation frequency $\Omega$; that is, we define

$$
\begin{aligned}
\sigma & =\sigma_{0}+\epsilon \sigma_{1}+\ldots \\
\xi & =\boldsymbol{\xi}^{0}+\epsilon \xi_{1}+\ldots
\end{aligned}
$$

where

$$
\epsilon=\Omega / \sigma_{0} .
$$


Here $\sigma$ is the oscillation frequency, and $\boldsymbol{\xi}$ is the displacement field. Inserting these definitions into equations (1) - (3) and equating powers of $\epsilon$ gives a hierarchical system of equations. The system of order $\epsilon^{0}$, by construction. is equivalent to that which describes a non-rotating neutron star (cf. McDermott et al. 1388).

To compute the rorational corrections, we expand the displacement $\xi^{1}$ in terms of the non-rotating basis functions $\xi^{0}$. That is.

$$
\xi^{1}=\sum_{j}\left(T_{j} \xi_{j}^{\mathrm{t}}+S_{j} \xi_{j}^{\mathrm{s}}\right)
$$

Here

$$
\xi_{j}^{s}=\left(C_{l}^{n}(r) Y_{l}^{m}(\theta, \phi), V_{l}^{n}(r) \frac{\partial Y_{l}^{m}(\theta, \phi)}{\partial \theta}, \frac{V_{l}^{n}(r)}{\sin \theta} \frac{\partial Y_{l}^{m}(\theta, \phi)}{\partial \phi}\right)
$$

are the spheroudal modes, and

$$
\boldsymbol{\xi}_{j}^{\ell}=\left(0, \frac{W_{l}^{n}(r)}{\sin \theta} \frac{\partial Y_{l}^{m}(\theta, \phi)}{\partial \phi},-W_{l}^{n}(r) \frac{\partial Y_{l}^{m}(\theta, \phi)}{\partial \theta}\right)
$$

are the toroidal modes. The index $j \equiv(l, m, n)$ represents the set of "quantum numbers" that defines a given spheroidal or toroidal mode. From the resulting first-order equations we can determine the corrections $\sigma_{1}, S_{i}$, and $T_{i}$.

For the present illustrative calculation we use a spherical unperturbed neutron star model (model NS05T7 from McDermott et al. 1988). In order to satisfy the slow rotation criterion, we require

$$
P \gg .545 \mathrm{msec} \frac{R_{6}^{3 / 2}}{\left(M / \mathrm{M}_{\odot}\right)^{1 / 2}},
$$

where $P$ is the rotation period of the neutron star. The first order corrections to the eigenfrequencies are, for toroidal modes,

$$
\sigma_{1}^{\mathrm{t}}=\frac{m \sigma_{0}}{l(l+1)}
$$

and for spheroidal modes,

$$
\sigma_{1}^{\mathrm{s}}=\frac{m \sigma_{0} \int_{0}^{R}\left[2 U_{l}^{n}(r) V_{l}^{n}(r)+\left(V_{l}^{n}(r)\right)^{2}\right] \rho_{0}(r) r^{2} d r}{\int_{0}^{R}\left[\left(U_{l}^{n}(r)\right)^{2}+l(l+1)\left(V_{l}^{n}(r)\right)^{2}\right] \rho_{0}(r) r^{2} d r}
$$

These results are in agreement with those of Pekeris et al. (1961), Backus and Gilbert (1961) and Unno et al. (1979). Note that the degeneracy of the non-rotating eigenfrequencies has been removed, as the corrections are proportional to the azimuthal order $m$. Table 1 contains the rotational corrections to the eigenfrequencies for several quadrupole modes of model NS05T7.

The corrected toroidal eigenfunctions, to first order in $\Omega$, are given by

$$
\xi_{l, m, n}^{\mathrm{ct}}=\xi_{l, m, n}^{\mathrm{l}}+\frac{\Omega}{\sigma_{0}} \sum_{n^{\prime}}\left(S_{m, n^{\prime}}^{l-1} \xi_{l-1, m, n^{\prime}}^{\mathrm{s}}+S_{m, n^{\prime}}^{l+1} \xi_{l+1, m, n^{\prime}}^{\mathrm{s}}\right)
$$

and the corrected spheroidal modes are similarly given by

$$
\xi_{l, m, n}^{\mathrm{cs}}=\xi_{l, m, n}^{\mathrm{s}}+\frac{\Omega}{\sigma_{0}} \sum_{n^{\prime}}\left[S_{m, n^{\prime}}^{l} \xi_{l, m, n^{\prime}}^{\mathrm{s}}+T_{m, n^{\prime}}^{l-1} \xi_{l-1, m, n^{\prime}}^{\mathrm{t}}+T_{m, n^{\prime}}^{l+1} \xi_{l+1, m, n^{\prime}}^{\mathrm{t}}\right] .
$$

Here the superscript (c) indicates a corrected function. The coefficients $S_{m, n^{\prime}}^{l-1}, S_{m, n^{\prime}}^{l+1}, S_{m, n^{\prime}}^{l}, T_{m, n^{\prime}}^{l-1}$ and $T_{m, n^{\prime}}^{l+1}$ are given in the Appendix. The restriction of the sums to include only corrections from the $l+1$, $l-1, l$ and like- $m$ functions is due to the angular integrals obtained from the first-order oscillation equations. 
Table 1 Rotational corrections to eigenfrequencies

\begin{tabular}{|crc|}
\hline Mode & $\sigma_{0}$ & $\epsilon \sigma_{1} / m \Omega$ \\
\hline${ }_{2} g_{3}^{s}$ & 14.32 & 0.1666 \\
${ }_{2} g_{2}^{s}$ & 17.06 & 0.1666 \\
${ }_{2} g_{1}^{s}$ & 27.04 & 0.1666 \\
${ }^{2} i_{1}$ & 69.34 & 0.1666 \\
${ }^{2} i_{2}$ & 918.7 & 0.1656 \\
${ }^{2} s_{1}$ & 2588.9 & 0.1192 \\
${ }_{2} s_{2}$ & 4650.8 & 0.1443 \\
${ }_{2} f$ & 15786.9 & 0.4957 \\
${ }_{2} p_{1}$ & 33798.7 & 0.0748 \\
${ }_{2} p_{2}$ & 39641.5 & 0.0430 \\
${ }_{2} t_{0}$ & 337.99 & 0.1666 \\
\hline
\end{tabular}

First-order rotational corrections to the eigenfrequencies of several quadrupole oscillation modes of model NS05T7. All frequencies are in Hertz. Here $1 g_{n}^{*}$ denotes surface $g$-modes, $l_{1}$ and $i_{2}$ the interfacial modes, $i s_{n}$ the shear modes, if the $f$-mode, and $i p_{n}$ the p-modes.

Partial Sums: surface g-modes $\theta=30$ degrees, $m=1, P P=1 \mathrm{sec}$

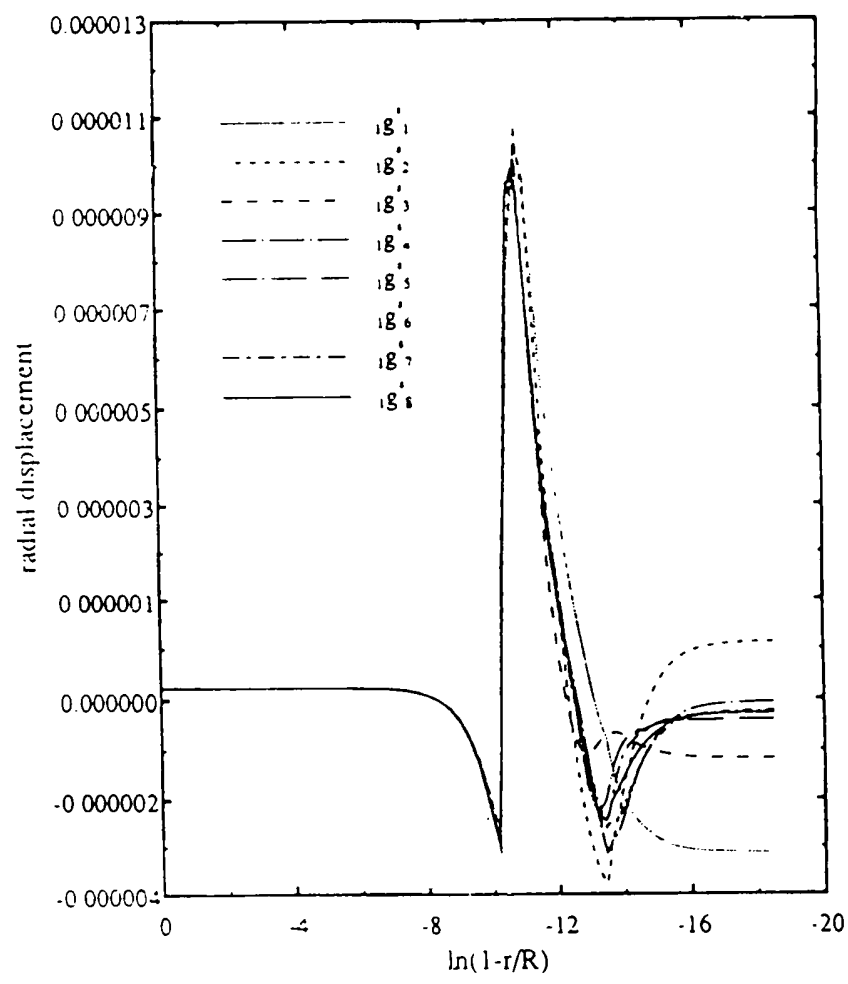

Figure 1a Correction sums for the ${ }_{2} t_{0}$ toroidal mode. The cumulative correction to the r-component of the displacement, due to the first eight $l=1$ surface $g$-mode overtones, is plotted as a function of $\ln (1-r / R)$. The $r$-component of the displacement is plotted at a constant value of $\theta=30^{\circ}$. The pulsar period was 0.1 seconds for this calculation. Note the alternating convergence of the overtone series.
Partial Sums: sg-modes $\theta=30, m=1, P P=1 \mathrm{sec}$

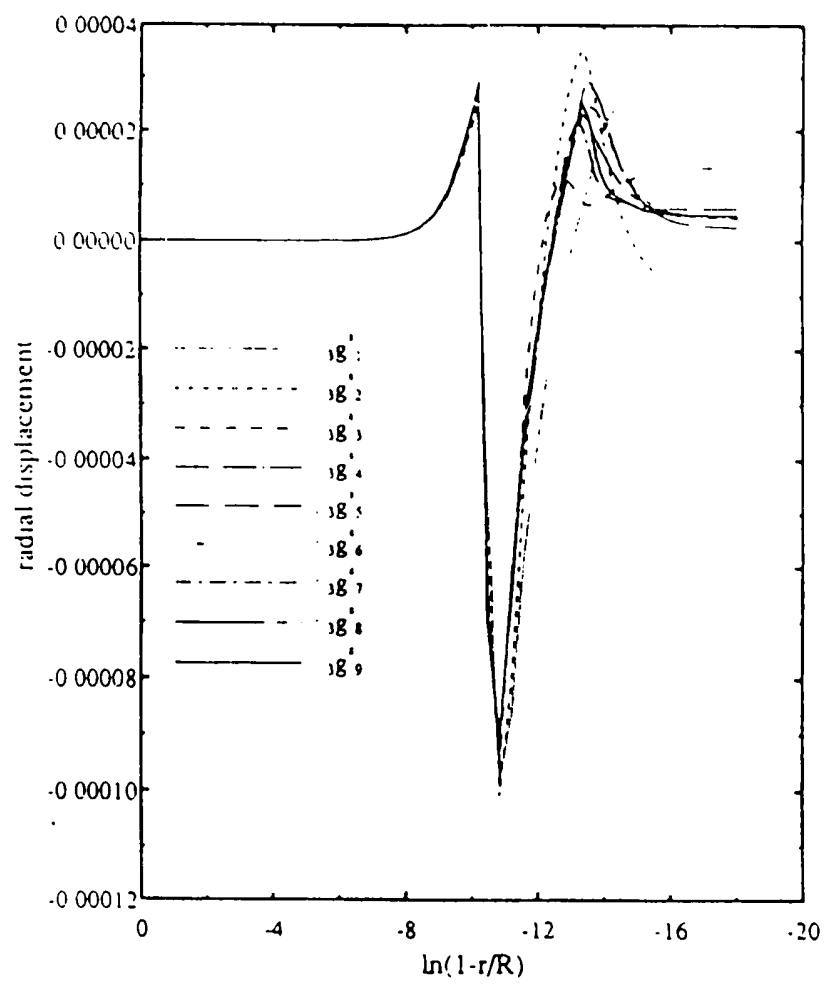

Figure $1 b$ Correction sums for the $2 t_{0}$ toroidal mode. The cumulative correction to the r-component of the displacement, due to the first nine $l=3$ surface $g$-mode overtones, is plotted as a function of $\ln (1-r / R)$.

\section{Results}

All calculations reported here were done with model NS05T7 studied by McDermott et al. (1988). This is a "soft"-equation-of-state model with a radius $R_{*}=9.839 \mathrm{~km}$, a mass $M_{*}=0.503 \mathrm{M}_{\odot}$, and a central 
temperature $1.03 \times 10^{7} \mathrm{~K}$. It is comprised of a fluid core, a solid crust, and a thin surface fluid "ocean". We use a Newton-Raphson relaxation method to compute the normal modes of this model. To compute the corrected eigenfunctions we must evaluate the sums in eqs.(13) and (14). We do this by computing the sum of the corrections due to each class of oscillation mode separately and then adding these contributions to obtain the total correction. The individual mode series are truncated when each sum has reached some specified level of convergence. Figure 1 shows the convergence properties of the correction sum, for the ${ }_{2} t_{0}$ mode, arising from surface $g$-modes. The number of overtones needed for convergence depends on the type of mode under consideration. The $p$-modes displayed the "slowest" convergence properties, whereas the surface $g$-modes converged at relatively low overtone numbers. The grid spacing of our equilibrium model sets an upper limit of about 50 to the number of overtones that we can accurately compute.

Corrected Eigenfunction: $2 \mathrm{t}_{0}$

Pulsar per $=01 \mathrm{sec}, m=1, \theta=30 \mathrm{deg}$

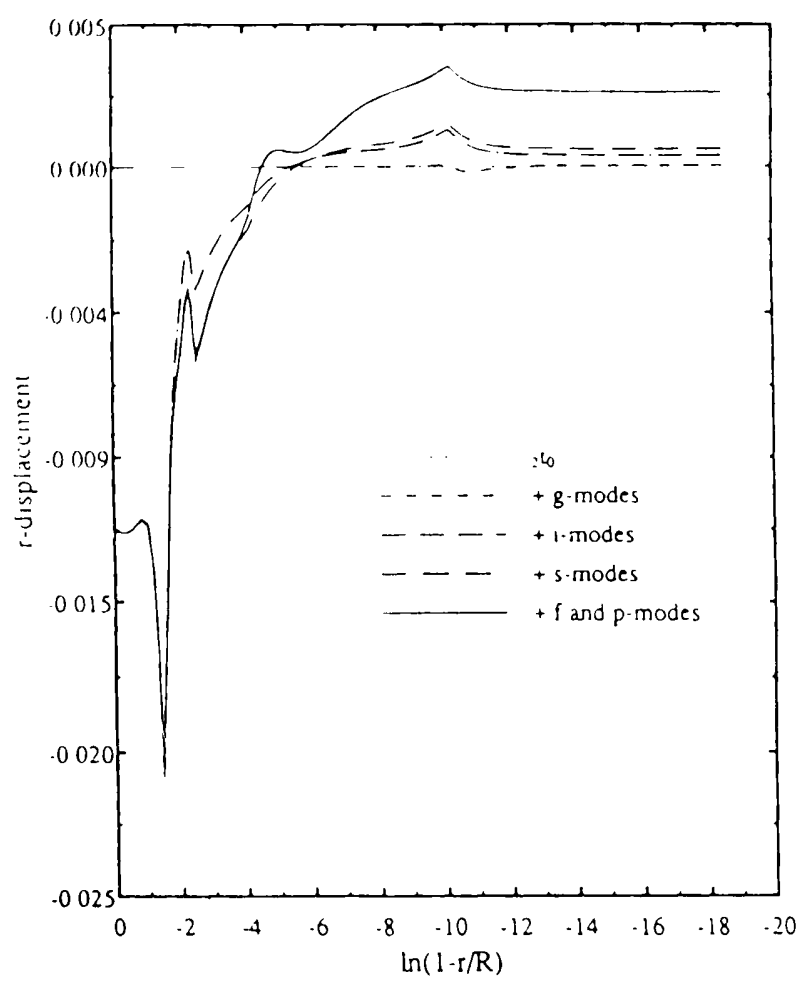

Figure 2a Plot of the cumulative correction to the $r$ component of the ${ }_{2} t_{0}$ toroidal mode eigenfunction due to each class of $l=1$ and $l=3$ spheroidal modes. The $r$ component of the uncorrected ${ }_{2} t_{0}$ eigenfunction is also plotted for comparison. The successive curves indicate the cumulative contributions from surface $g$-modes $\left(, g_{n}^{*}\right)$, intetfacial modes $\left({ }_{1} i_{1}\right.$ and $\left.l_{l} i_{2}\right)$, shear modes $\left(l_{n}\right)$, and the $f$ and $p-$ modes ( $f$ and $\left(p_{n}\right)$. The displacement is shown for $\theta=30^{\circ}$. The pulsar period was 0.1 seconds for this calculation.
Corrected Eigenfunction: ${ }_{2} t_{0}$ Pulsar per $=0.1 \mathrm{sec}, m=1, \theta=30 \mathrm{deg}$

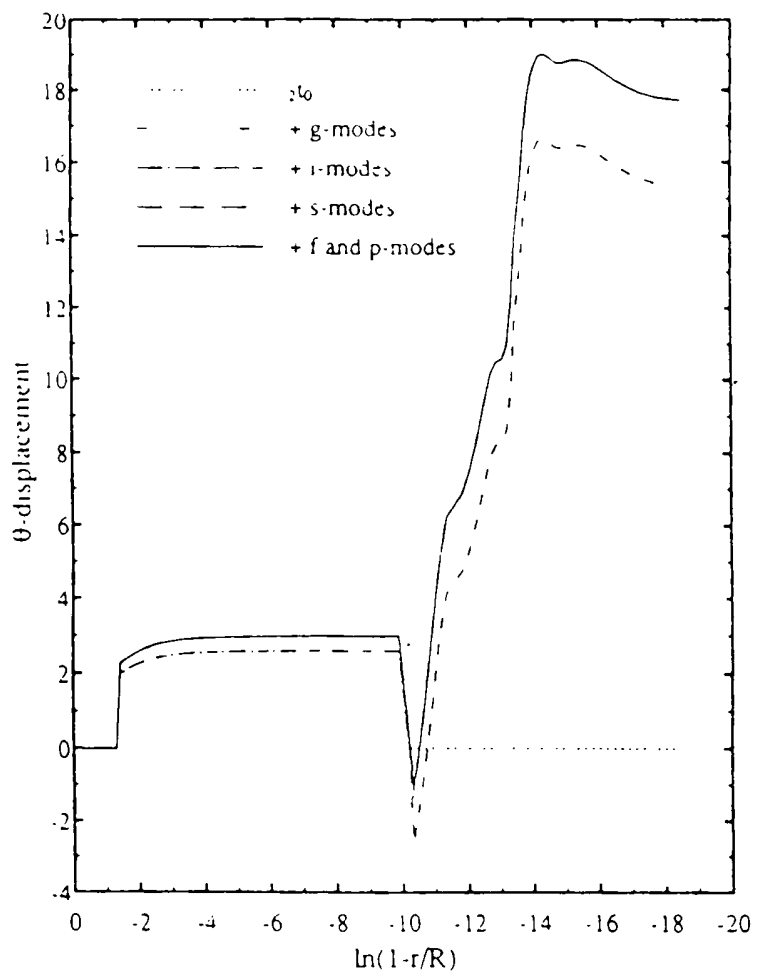

Figure 2b Plot of the cumulative correction to the $\theta$ component of the ${ }_{2} t_{0}$ toroidal mode eigenfunction. Other details are the same as in figure $2 \mathrm{a}$. Notice the large transverse displacement that is now present at the surface of the star. This is due to the coupling with surface $g$-modes, which have large transverse displacements in the surface fluid "ocean".

Figure 2 shows plots of the corrections to the ${ }_{2} t_{0}$ toroidal mode eigenfunction due to each class of spheroidal mode, as well as the fully corrected eigenfunctions. The uncorrected modes are also plotted for comparison. The three parts to the figure (a), (b), and (c) show the $r$-component, $\theta$-component, and the $\phi$-component respectively of the displacement $\xi_{2,1,0}^{\mathrm{ct}}$. Notice the non-zero radial displacement produced by the spheroidal coupling, and that the toroidal eigenfunctions are no longer confined to the crust of the star. The coupling with the spheroidal modes (principally the surface $g$-modes) produces a significant transverse displacement at the surface of the star. This may enable energy associated with a crust-quake (glitch) to be channeled into surface motions, where it might produce potentially observable effects in the pulsar emissinn process or a $\gamma$-ray burst event.

We have used these results to compute the neutrino damping rate, in the quasi-adiabatic approximation (Cox 1980), for the ${ }_{2} t_{0}$ and ${ }_{2} t_{1}$ toroidal modes. The neutrino damping times thus calculated for model NS05T7 are significantly longer than the gravitational radiation damping times computed by McDermott et al. (1988); however, the extreme temperature sensitivity of the neutrino emissivity indicates 
Corrected Eigenfunction: ${ }_{2} t_{0}$

Pulsar per. $=0.1 \mathrm{sec}, m=1, \theta=30 \mathrm{deg}$

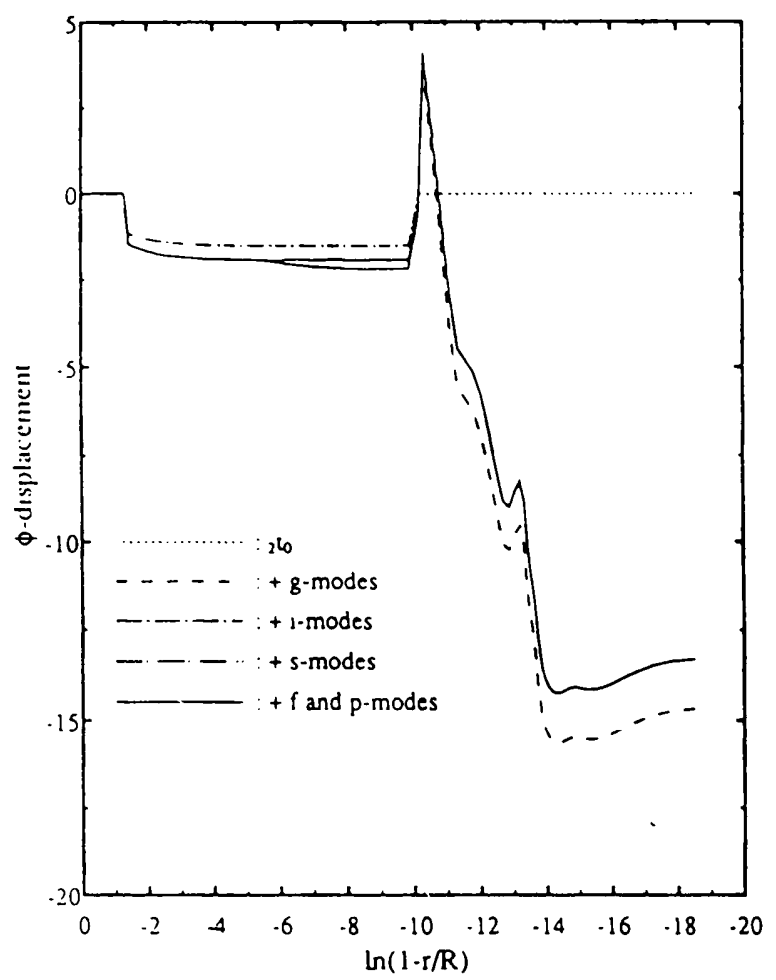

Figure 2c Plot of the cumulative correction to the $\phi$-component of the ${ }_{2} t_{0}$ toroidal mode eigenfunction. Other details are the same as in figure $2 a$.

that toroidal oscillations of hotter models should be damped by neutrino emission on timescales comparable to the gravitational radiation decay time. A simple scaling argument based on the temperature dependence of the dominant neutrino reactions, and the results of our calculations for model NS05T7 gives

$$
\tau_{\nu} \approx \tau_{0}(P / 1 \mathrm{sec})^{2}\left(10^{7} \mathrm{~K} / T_{\mathrm{c}}\right)^{7},
$$

where $\tau_{0}=1.0 \times 10^{15}$ years, for the ${ }_{2} t_{0}$ mode, $T_{c}$ is the central temperature of the neutron star, and $P$ is the rotation period.

\section{Conclusions}

We have computed the first-order rotational corrections to the oscillation frequencies and eigenfunctions of a realistic neutron-star model. The presence of the Coriolis force couples $l=l_{0}$ oscillation modes to $l=l_{0} \pm 1$ modes. This coupling has interesting consequences for the damping of toroidal oscillations, via neutrino emission, in a rotating neutron star. The rotationally corrected toroidal eigenfunctions are no longer confined to the crust, and can have appreciable displacements at the surface of the star. We intend to use this work in further studies of the interaction between an oscillating neutron star and its surroundings.

\section{Appendix}

The correction coefficients for the toroidal modes are

$$
S_{m, n^{\prime}}^{l-1}=-\frac{2 i \sigma_{t(l, m, n)} \sigma_{0}(l+1)(l+m) \int_{0}^{R} W_{l}^{n}\left(U_{l-1}^{n^{\prime}}-(l-1) V_{l-1}^{n^{\prime}}\right) \rho_{0} r^{2} d r}{(2 l+1)\left(\sigma_{t(l, m, n)}^{2}-\sigma_{\iota\left(l-1, m, n^{\prime}\right)}^{2}\right) \int_{0}^{R}\left[\left(U_{l-1}^{n^{\prime}}\right)^{2}+l(l-1)\left(V_{l-1}^{n^{\prime}}\right)^{2}\right] \rho_{0} r^{2} d r}
$$


and

$$
S_{m n^{\prime}}^{l+1}=\frac{2 i \sigma_{t(l, m, n)} \sigma_{0} l(l-m+1) \int_{0}^{R} W_{l}^{n}\left(U_{l+1}^{n^{\prime}}+(l+2) V_{l+1}^{n^{\prime}}\right) \rho_{0} r^{2} d r}{(2 l+1)\left(\sigma_{t(l, m, n)}^{2}-\sigma_{\bullet\left(l+1, m, n^{\prime}\right)}^{2}\right) \int_{0}^{R}\left[U_{l+1}^{n^{\prime} 2}+(l+1)(l+2) V_{l+1}^{n^{\prime} 2}\right] \rho_{0} r^{2} d r} .
$$

For the corrections to spheroidal modes we have

$$
\begin{gathered}
S_{m, n^{\prime}}^{l}=\frac{2 m \sigma_{\bullet} \sigma_{\bullet}(l, m, n) \int_{0}^{R}\left[V_{l}^{n} C_{l}^{n^{\prime}}+U_{l}^{n} V_{l}^{n^{\prime}}+V_{l}^{n} V_{l}^{n^{\prime}}\right] \rho_{0} r^{2} d r}{\left(\sigma_{a(l, m, n)}^{2}-\sigma_{o\left(l, m, n^{\prime}\right)}^{2}\right) \int_{0}^{R}\left[\left(U_{l}^{n^{\prime}}\right)^{2}+l(l+1)\left(V_{l}^{n^{\prime}}\right)^{2}\right] \rho_{0} r^{2} d r}, \\
T_{m, n^{\prime}}^{l-1}=\frac{2 i \sigma_{s(l, m, n)} \sigma_{\bullet}(l+m) \int_{0}^{R} W_{l-1}^{n^{\prime}}\left(C_{l}^{n}+(l+1) V_{l}^{n}\right) \rho_{0} r^{2} d r}{l(2 l+1)\left(\sigma_{s(l, m, n)}^{2}-\sigma_{t\left(l-1, m, n^{\prime}\right)}^{2}\right) \int_{0}^{R}\left(W_{l-1}^{n^{\prime}}\right)^{2} \rho_{0} r^{2} d r},
\end{gathered}
$$

and

$$
T_{m, n^{\prime}}^{l+1}=\frac{2 i \sigma_{s(l, m, n)} \sigma_{.}(l-m+1) \int_{0}^{R} W_{l+1}^{n^{\prime}}\left(l V_{l}^{n}-U_{l}^{n}\right) \rho_{0} r^{2} d r}{(l+1)(2 l+1)\left(\sigma_{o(l, m, n)}^{2}-\sigma_{t\left(l+1, m, n^{\prime}\right)}^{2}\right) \int_{0}^{R}\left(W_{l+1}^{n^{\prime}}\right)^{2} \rho_{0} r^{2} d r}
$$

Here $U_{k}^{n}$ and $V_{k}^{n}$ are the spheroidal mode eigenfunctions, and $W_{k}^{n}$ is the toroidal eigenfunction [cf. eqs.(8) and (9)].

Acknowledgments: This work has been supported by the National Science Foundation under grant AST 88-20322 through the University of Rochester, and in part by NASA under grant NSG-7562 also through the University of Rochester. 